\title{
Vaginal Cuff Brachytherapy
}

National Cancer Institute

\section{Source}

National Cancer Institute. Vaginal Cuff Brachytherapy. NCI Thesaurus. Code C158398.

Radiation delivered by a radiation source contained in an intra-vag inal cylinder to target neoplasms of the vaginal cuff. 